\title{
Preface: Advances in post-processing and blending of deterministic and ensemble forecasts
}

\author{
Stephan Hemri ${ }^{1,2}$, Sebastian Lerch ${ }^{3}$, Maxime Taillardat $^{4}$, Stéphane Vannitsem ${ }^{5,6}$, and Daniel S. Wilks ${ }^{7}$ \\ ${ }^{1}$ Federal Office of Meteorology and Climatology, MeteoSwiss, Operation Center 1, 8058 Zurich Airport, Switzerland \\ ${ }^{2}$ Institute of Mathematics, University of Zurich, Winterthurerstrasse 190, 8057 Zurich, Switzerland \\ ${ }^{3}$ Institute for Stochastics, Karlsruhe Institute of Technology, Englerstr. 2, 76131 Karlsruhe, Germany \\ ${ }^{4}$ Météo-France, CNRM UMR 3589, Toulouse, France \\ ${ }^{5}$ Royal Meteorological Institute of Belgium, Avenue Circulaire 3, 1180 Brussels, Belgium \\ ${ }^{6}$ European Meteorological Network (EUMETNET), Avenue Circulaire 3, 1180 Brussels, Belgium \\ ${ }^{7}$ Earth and Atmospheric Sciences, Cornell University, Ithaca, NY, USA
}

Correspondence: Stéphane Vannitsem (svn@meteo.be)

Received: 14 October 2020 - Published: 17 November 2020

\begin{abstract}
The special issue on advances in post-processing and blending of deterministic and ensemble forecasts is the outcome of several successful successive sessions organized at the General Assembly of the European Geosciences Union. Statistical post-processing and blending of forecasts are currently topics of important attention and development in many countries to produce optimal forecasts. Ten contributions have been received, covering key aspects of current concerns on statistical post-processing, namely the restoration of inter-variable dependences, the impact of model changes on the statistical relationships and how to cope with it, the operational implementation at forecasting centers, the development of appropriate metrics for forecast verification, and finally two specific applications to snow forecasts and seasonal forecasts of the North Atlantic Oscillation.
\end{abstract}

Statistical post-processing of weather, seasonal and decadal forecasts is key to optimizing the information provided by dynamical forecasting systems. Many weather, hydrological and climate centers worldwide are developing postprocessing systems in order to substantially reduce forecast errors (Vannitsem et al., 2018, 2020; Wilks, 2019). Strategies are however very different from one center to another, due to the specific priorities of each center. These priorities depend on the atmospheric or climate fields requested by end users, the forecasts available at the center, the computational resources, and the availability of past datasets upon which the statistical relationships can be trained. The resulting statistical post-processing techniques are accordingly highly diverse, as illustrated in the papers in this special issue. This issue has been organized following the success of the annual sessions on statistical post-processing organized as part of the General Assembly of the European Geosciences Union.

Originally, post-processing was developed to correct single deterministic forecasts (Glahn and Lowry, 1972). In the last 30 years, important developments have been put forward by the weather centers providing global forecasts, in order to also provide information about forecast uncertainty. Quantification of forecast uncertainty has been approached primarily through the development of ensemble forecasts, in which a collection of forecasts is started from different initial conditions and, more recently, also using stochastic (or different) physical parameterizations (Buizza, 2018). These ensembles can be used to formulate probabilistic forecasts, although in raw form they are generally found to be biased and overconfident. Therefore, corrections should be provided, and diverse strategies have been developed to do so. The papers in this special issue describe some of the particular ways that this problem has been addressed.

Two main approaches have been developed for postprocessing ensemble forecasts: techniques that apply corrections to individual ensemble members and techniques that specify probability distributions for quantities of interest. In the former case, the idea is to keep track of the spatiotemporal evolution of the fields produced by the underlying dy- 
namical model and to maintain as far as possible the intervariable and spatial dependences inherited from it, although some random errors may not be properly accounted for. For the latter, the idea is to take into account all possible sources of error and to provide a calibrated ("reliable") probabilistic forecast, but this is often at the expense of destroying correlation structures across time, space, and/or different forecast variables.

The paper of Lerch et al. (2020) reviews techniques used to preserve or correct the inter-variable dependences and spatiotemporal coherence of probabilistic forecasts. This question is also addressed by Steinheuer and Friederichs (2020) for the specific application of providing and correcting the wind gust forecasts generated by a regional model at any height in the atmosphere, where the Pickands' bivariate dependence function is used to produce realistic dependencies of wind gusts in the vertical.

Information on past forecasts and observations is required in order to develop appropriate statistical post-processing schemes. Ideally the training forecasts should be produced by a specific, unchanging model version that continues to be used in practice. In an operational setting, however, the dynamical models change regularly as improvements to the dynamics, the physics, or the numerics are introduced. There are possible strategies to ameliorate the basic problem of mismatch between the training data and the current operational version. One, demonstrated by Lang et al. (2020), consists of developing time-adaptive training schemes derived from the sliding-window approach, which reduces the impact of a model change by progressively mixing old forecasts with new ones. Several strategies are investigated. Another way to deal with this problem is discussed by Demaeyer and Vannitsem (2020), who describe the use of linear response theory. The idea is to evaluate, before the model change, the impact of this change on the statistics of the post-processing method. The response of these statistics to a model change can be inferred using a linearized version of the model before the change.

The operational implementation of statistical postprocessing imposes time-critical constraints so that the complexity of post-processing algorithms must be balanced against the need to reasonably limit the computational requirements. This issue is especially relevant for forecasts with high spatiotemporal resolution, requiring the use of very detailed models and huge output files. We enter here into the domain of "big data". The post-processing techniques should be able to cope with new types of high-resolution products and, at the same time, be highly efficient. These challenges are discussed by Hess (2020) in which the post-processing suite of the German national weather service (Deutscher Wetterdienst) is described. This suite uses relatively simple post-processing techniques, with a specific focus on the correction of extreme events. A second operational system is described by Taillardat and Mestre (2020), who use a tool from the world of machine learning: quantile regression forests.
These approaches are very successful in dealing with the large sets of data associated with high-resolution forecasting, in particular for precipitation and temperature, but clever strategies to reduce the required computer time are needed. Finally, optimal use of the forecast errors in earlier projections can be used to reduce subsequent errors in the same forecast trajectory. This promising technique, named RAFT (rapid adjustment of forecast trajectories), is presented by Schuhen (2020).

A key step in the development of statistical postprocessing schemes is evaluation of the quality of the forecasts. This process depends critically on the metrics used, as discussed in detail by Thorarinsdottir and Schuhen (2018). Usually, univariate metrics are used. Jacobson et al. (2020) propose use of metrics that take into account the spatial properties of the fields of interest. They propose a new diagnostic tool called the fraction of threshold exceedance, which allows projection of possibly high-dimensional multivariate fields onto a single univariate quantity, which can then be analyzed with standard metrics.

Finally, two diverse applications of statistical postprocessing in less common settings have been addressed in this special issue. First, the prediction of snow depth for early warning of avalanches, road viability and ski resort management is described by Nousu et al. (2019), who emphasize the need for long reforecast datasets in order to provide sufficient sample size to represent the various situations that could be encountered in mountainous regions. A second application, to the seasonal forecasting of the North Atlantic Oscillation, is made by Düsterhus (2020) using statistical resampling of deterministic forecasts together with their uncertainties.

\section{References}

Buizza, R.: Ensemble forecasting and the need for calibration, in: Statistical postprocessing of ensemble forecasts, edited by: Vannitsem, S., Wilks, D. S., and Messner, J. W., chap. 2, 15-48, Elsevier, Amsterdam, the Netherlands, https://doi.org/10.1016/B9780-12-812372-0.00002-9, 2018.

Demaeyer, J. and Vannitsem, S.: Correcting for model changes in statistical postprocessing - an approach based on response theory, Nonlin. Processes Geophys., 27, 307-327, https://doi.org/10.5194/npg-27-307-2020, 2020.

Düsterhus, A.: Seasonal statistical-dynamical prediction of the North Atlantic Oscillation by probabilistic post-processing and its evaluation, Nonlin. Processes Geophys., 27, 121-131, https://doi.org/10.5194/npg-27-121-2020, 2020.

Glahn, H. R. and Lowry, D. A.: The Use of Model Output Statistics (MOS) in Objective Weather Forecasting, J. Appl. Meteorol., 11, 1203-1211, 1972.

Hess, R.: Statistical postprocessing of ensemble forecasts for severe weather at Deutscher Wetterdienst, Nonlin. Processes Geophys., 27, 473-487, https://doi.org/10.5194/npg-27-473-2020, 2020.

Jacobson, J., Kleiber, W., Scheuerer, M., and Bellier, J.: Beyond univariate calibration: verifying spatial structure in ensembles 
of forecast fields, Nonlin. Processes Geophys., 27, 411-427, https://doi.org/10.5194/npg-27-411-2020, 2020.

Lang, M. N., Lerch, S., Mayr, G. J., Simon, T., Stauffer, R., and Zeileis, A.: Remember the past: a comparison of time-adaptive training schemes for non-homogeneous regression, Nonlin. Processes Geophys., 27, 23-34, https://doi.org/10.5194/npg-27-232020, 2020.

Lerch, S., Baran, S., Möller, A., Groß, J., Schefzik, R., Hemri, S., and Graeter, M.: Simulation-based comparison of multivariate ensemble post-processing methods, Nonlin. Processes Geophys., 27, 349-371, https://doi.org/10.5194/npg-27-349-2020, 2020.

Nousu, J.-P., Lafaysse, M., Vernay, M., Bellier, J., Evin, G., and Joly, B.: Statistical post-processing of ensemble forecasts of the height of new snow, Nonlin. Processes Geophys., 26, 339-357, https://doi.org/10.5194/npg-26-339-2019, 2019.

Schuhen, N.: Order of operation for multi-stage post-processing of ensemble wind forecast trajectories, Nonlin. Processes Geophys., 27, 35-49, https://doi.org/10.5194/npg-27-35-2020, 2020.

Steinheuer, J. and Friederichs, P.: Vertical profiles of wind gust statistics from a regional reanalysis using multivariate extreme value theory, Nonlin. Processes Geophys., 27, 239-252, https://doi.org/10.5194/npg-27-239-2020, 2020.

Taillardat, M. and Mestre, O.: From research to applications - examples of operational ensemble post-processing in France using machine learning, Nonlin. Processes Geophys., 27, 329-347, https://doi.org/10.5194/npg-27-329-2020, 2020.
Thorarinsdottir, T. L. and Schuhen, N.: Verification: assessment of calibration and accuracy, in: Statistical postprocessing of ensemble forecasts, edited by: Vannitsem, S., Wilks, D. S., and Messner, J. W., chap. 6, 155-186, Elsevier, Amsterdam, the Netherlands, https://doi.org/10.1016/b978-0-12812372-0.00006-6, 2018.

Vannitsem, S., Wilks, D. S., and Messner, J. (Eds): Statistical Postprocessing of Ensemble Forecasts, Elsevier, Amsterdam, the Netherlands, 2018.

Vannitsem, S., Bremnes, J. B., Demaeyer, J., Evans, G. R., Flowerdew, J., Hemri, S., Lerch, S., Roberts, N., Theis, S., Atencia, A., Ben Bouallègue, Z., Bhend, J., Dabernig, M., De Cruz, L., Hieta, L., Mestre, O., Moret, L., Odak Plenković, I., Schmeits, M., Taillardat, M., Van den Bergh, J., Van Schaeybroeck, B., Whan, K., and Ylhaisi, J.: Statistical Postprocessing for Weather Forecasts - Review, Challenges and Avenues in a Big Data World, Bulletin of the American Meteorological Society, in press, 2020.

Wilks, D. S.: Statistical Methods in the Atmospheric Sciences, 4th edn., Academic Press, Amsterdam, the Netherlands, 2019. 